\title{
Recovery of Neurological Functions in Non-Human Primate Model of Parkinson's Disease by Transplantation of Encapsulated Neonatal Porcine Choroid Plexus Cells
}

\author{
Xian-Ming Luo ${ }^{\mathrm{a}}$, Hai Lin ${ }^{\mathrm{b}}$, Wei Wang ${ }^{\mathrm{a}, *}$, Marilyn S. Geaney ${ }^{\mathrm{b}}$, Lee Law $^{\mathrm{b}}$, Shaun Wynyard ${ }^{\mathrm{b}}$, \\ Shamim B. Shaikh ${ }^{\mathrm{b}}$, Henry Waldvogel ${ }^{\mathrm{c}}$, Richard L.M. Faull ${ }^{\mathrm{c}}$, Robert B. Elliott ${ }^{\mathrm{b}}$, \\ Stephen J.M. Skinner ${ }^{\mathrm{d}}$, Jacqueline E. Lee ${ }^{\mathrm{b}}$ and Paul L.-J. Tan ${ }^{\mathrm{b}, *}$ \\ ${ }^{\mathrm{a}}$ The Third Xianya Hospital of Central-South University, Changsha, China \\ ${ }^{\mathrm{b}}$ Living Cell Technologies Limited, Papatoetoe, Auckland, New Zealand \\ ${ }^{\mathrm{c}}$ School of Medical Sciences, University of Auckland, Auckland, New Zealand \\ ${ }^{\mathrm{d}}$ Department of Physiology, University of Auckland, Auckland, New Zealand
}

\begin{abstract}
Parkinson's disease (PD) is a neurodegenerative disease that is primarily characterized by degeneration of dopaminergic (DA) neurons in the substantia nigra (SN) and a loss of their fibre projections in the striatum. We utilized the neonatal porcine choroid plexus (CP), an organ that secretes cerebrospinal fluid containing various types of neurotrophic and neuroprotective factors, to ameliorate the Parkinsonian symptoms in MPTP (1-methyl-4-phenyl-1,2,3,6-tetrahydropyridine)-treated rhesus monkeys without requiring immunosuppression. We demonstrate that transplanted encapsulated CP clusters (eCPs) significantly improved neurological functions in MPTP-treated monkeys during the course of six months after transplantation $(p<0.001)$ when compared with monkeys implanted with empty capsules or subjected to sham surgery. The improvement in neurological scores was accompanied by a corresponding improvement in apomorphine-induced circling behaviour $(p<0.001)$ as well as increased tyrosine hydroxylase $(\mathrm{TH})$ staining in the striatum. Our results suggest that eCPs are a promising cell therapeutic agent to treat Parkinson's disease.
\end{abstract}

Keywords: Parkinson's disease (PD), MPTP, apomorphine, monkey, choroid plexus (CP), encapsulated choroid plexus clusters (eCPs), neurotrophin, substantia nigra (SN), striatum, putamen, TH fibre, encapsulation, motor control, neurological recovery, dopaminergic, tyrosine hydroxylase

ABBREVIATIONS
PD Parkinson's disease
CP Choroid plexus cells
eCP encapsulated chroid plexus cluster

${ }^{*}$ Correspondence to: Paul L.-J. Tan, Living Cell Technologies Limited, Papatoetoe, Auckland, New Zealand. E-mail: ptan@1ctglobal.com; Wei Wang, The Third Xianya Hospital of Central-South University, Changsha, China. Tel.: +64 9270 7948; Fax: +64 9276 2691; E-mail: wawe01cn@yahoo.com.cn.

\author{
NT Neurotrophin \\ DA Dopaminergic \\ SN Substantia Nigra \\ MPTP 1-methyl-4-phenyl-1,2,3,6-tetrahydropyridine \\ MPP+ 1-methyl-4-phenylpyridinium \\ TH Tyrosine hydroxylase \\ AIC Apomorphine-induced circling \\ SM Sensorimotor (territory of the striatum) \\ Assoc Associative (territory of the striatum) \\ Lim Limbic (territory of the striatum)
}




\section{INTRODUCTION}

The Choroid plexus (CP) produces cerebrospinal fluid containing several essential factors that maintain homeostasis of the brain and protect it from toxic insults [1-5]. We have previously shown by gene expression array analysis that the porcine $\mathrm{CP}$ expresses genes encoding a variety of growth factors and neurotrophins as well as proteins with anti-oxidant and chaperone activities [6]. Further analyses of the neonatal porcine $\mathrm{CP}$ clusters in culture have revealed that many neurotrophins and chaperone proteins are indeed secreted by these cells at physiological levels [6].

We have investigated whether implants of CP cells may be used to ameliorate various neurological diseases in animal models. To protect the CP cells from the xenogenic response of the host immune system, we encapsulate porcine $\mathrm{CP}$ clusters (eCPs) in an alginate microcapsule to provide an immunobarrier [6-10]. In a rat stroke model using a one-hour middle cerebral artery occlusion/reperfusion, we demonstrated that an immediate post-lesion placement of porcine eCPs significantly reduced motor asymmetry and neurological impairment as well as striatal infarction volume [7]. We have also shown that neonatal porcine eCPs were able to reduce striatal lesion in both rat and monkey models of Huntington's Disease [8, 9]. Finally, in a rat model of PD generated by unilateral injection of 6-hydroxydopamine, eCPs transplanted at four weeks after introducing the lesion improved the amphetamineinduced circling behaviour and increased tyrosine hydroxylase staining in the striatum [10]. Collectively, we have demonstrated that neonatal porcine eCPs provide therapeutic levels of neuroprotective and neurorestorative effects to elicit significant neurologic improvement in various animal models of brain diseases.

In the current study, we investigate whether eCPs from neonatal pigs can protect dopaminergic (DA) neurons and their fibres from degeneration or promote their regeneration in a non-human primate model of $\mathrm{PD}$. DA neurons in the substantia nigra $(\mathrm{SN})$, whose loss is particularly pronounced in $\mathrm{SN}$ compacta $(\mathrm{SNc})$ of PD patients, normally project to the striatum that includes the putamen, caudate nucleus and nucleus accumbens $[11,12]$. The striatum, which also receives inputs from other areas of the brain, is divided into three anatomic and functional territories that are confirmed in behavioural studies: the sensorimotor (SM) territory which receives inputs from sensory and motor cortex, associative (Assoc) territory which receives input mainly from the prefrontal cortex, and limbic (Lim) territory which receives inputs from the limbic nuclei amygdala $[11,12]$. In both PD and neurotoxin MPTP -treated animals, the striatal dopaminergic input is profoundly affected in the sensorimotor territory followed by the associative territory while that in the limbic territory is mostly resistant to degeneration [13-18]. In both conditions, post-mortem analysis revealed a severe dopamine depletion in the putamen while the caudate was relatively spared $[17,18]$.

We report that only the animals that were treated with porcine eCPs in our study exhibited a significant neurological score improvement by $39.2 \%(p<0.001)$ while those in the Control and Sham groups did not. In parallel, while all monkeys exhibited apomorphinestimulated asymmetrical turns, only the eCP-treated group showed a significant $60.6 \%$ reduction in the number of turns following implant surgery $(p<0.001)$. Post-mortem examination of the TH-stained fibre density in both the associative and sensorimotor territories of the striatum suggested that one of the means by which neonatal porcine CPs improve neurological functions in the non-human primate model of PD involves restoring $\mathrm{TH}$-positive fibre networks in the striatum.

\section{MATERIALS AND METHODS}

\section{Encapsulated chroid plexus preparation from neonatal pigs}

All animal work performed at LCT was approved by the Animal Ethics Committee under the direction of the National Animal Ethics Advisory Council of New Zealand. Neonatal Auckland Island Pigs were bred and the tissues harvested by trained staff at Living Cell Technologies (LCT) Inc (Auckland, NZ) using protocols approved by the LCT Animal Ethics Committee under the Guidelines of the National Animal Ethics Advisory Council of New Zealand. Neonatal porcine choroid plexus cells were surgically removed from new born (6-15 days old) Auckland Island strain piglets under aseptic conditions. The $\mathrm{CP}$ clusters were prepared and maintained in culture for seven days after which they were encapsulated within alginate-polyornithine capsules $(600-660 \mu \mathrm{m}$ in diameter) as described previously [6-10]. The bioactivity of CPs and eCPs were confirmed by measuring their secretion of VEGF and BDNF into media using respective ELISA kits according to the suppliers instructions (Quantikine, MN USA; Promega, WI, USA) as described previously [6]. 


\section{Rhesus monkeys}

Fifteen male rhesus monkeys (Macaca mulatta), of average age of 8 years and of average body weight $(8.5 \mathrm{~kg})$, were used in the experiment (See Suppl. Material $\mathrm{S} 1)$. Monkeys were individually housed in stainless steel, slat floor cages. Study rooms were maintained on a 12-hour light/dark cycle, within a temperature range of $18^{\circ} \mathrm{C}$ to $28^{\circ} \mathrm{C}$, and a relative humidity range of 30 to $70 \%$. Kennel King Professional Lab-Primates Feed (Foshan Taierfu Pets Feed Company Ltd) was provided daily in amounts appropriate for the size and age of the animals. This diet was also supplemented with fruits or vegetables 2-3 times weekly. Water was provided ad libitum by an automatic watering system. Animals were observed daily by veterinary staff experienced with the normal care, handling and behaviour of primates. The experiment was approved by Animal Ethics Committee of Central-South University, Changsha, China.

\section{MPTP lesion induction}

Rhesus monkeys were infused with MPTP via the right internal carotid artery to produce a lesion in the nigrostriatal pathway following an established protocol at Central-South University, Changsha, China [19] and by others [20, 21]. A catheter was inserted into the proximal end of the right internal carotid artery through a small incision in the skin under the guidance of angiography. MPTP was infused through the catheter at a dose of $1.5 \mathrm{mg} / \mathrm{kg}$ (dissolved in $50 \mathrm{ml} 38^{\circ} \mathrm{C}$ saline immediately before use) at a rate of $2 \mathrm{ml} / \mathrm{min}$. Routine hemostasis and suturing methods were used after the MPTP infusion. Animals were closely monitored for recovery and returned to their cages after they were fully awake. After four weeks of measuring behavioural scores using a rating scale used by Takagi et al. [22], two spontaneously recovered animals were excluded from the study and all fifteen remaining animals verified to have stable lesions, as demonstrated by displaying steady neurological score deficits and asymmetric turning movements following apomorphine challenge, were included in further study.

\section{Implantation surgery}

The caudate nucleus of the striatum was chosen as an implantation site because it is readily identified from cross-section images under Computed Tomography $(\mathrm{CT})$ transaxial scanning (Somatom Balance,
Siemens, Germany) due to its position near the ventricle. Between week eight and week twelve after MPTP treatment, the caudate nucleus of the monkeys was implanted with 40 capsules containing choroid plexus cells (Treated, $n=6$ ) or with empty capsules (Control, $n=6$ ). In sham operated monkeys (Sham, $n=3$ ), catheters were introduced into the MPTP-lesioned brains but no capsules were implanted. Monkeys were anesthetized with ketamine $(10 \mathrm{mg} / \mathrm{kg})$. When fully anesthetized, the heads were fixed in a primate stereotaxic frame. The 3D coordinates of the implantation site were calculated based on the CT images. Immediately above the caudate nucleus on the right side of the skull two holes $(6 \mathrm{~mm}$ apart) were drilled at precise stereotactic co-ordinates and the dura was punctured under the guidance of CT. Two catheters, each loaded with 20 capsules, were inserted into the caudate nucleus via the drilled holes, guided by a Computed Axial Tomography (CAT) scan. Capsules were implanted into the caudate nucleus after confirmation that the tip of catheter was in the correct position according to the CT scan.

\section{Neurological scores assessment}

The neurological scoring system used by Takagi et al. [22] was used throughout the study (Table 1). Two independent observers blinded to the treatment options assessed the animals. A behavioral assessment was made before MPTP administration in order to exclude any animals with spontaneous PD symptoms that may have been caused by ageing or other causes. Neurological scores were determined 4 weeks post lesion (baseline) and at 2, 4, 8, 12, 16 and 24 weeks post-implantation.

\section{Asymmetric rotation}

There was no indication that nigrostriatal capsule implants in monkey brains caused any obvious discomfort or unusual behaviour at rest. PD Monkeys were challenged with apomorphine $(0.2 \mathrm{mg} / \mathrm{kg}, \mathrm{i} . \mathrm{m}$. $)$ at 4 weeks post lesion (baseline) and 2, 4, 8, 12, 16 and 24 weeks post capsule implant or sham surgery to elicit apomorphine-induced circling (AIC) behaviour [23]. The AIC score at 4 weeks post-lesion was used to confirm that animals had achieved the required minimum response of 7 turns per minute as the standard of an adequate lesion. Asymmetric rotations were recorded for one hour by video camera, the recordings blind-coded and the turning responses evaluated by two independent trained observers at a later date. Some of the 
evaluations were randomly chosen for auditing by two other trained staff.

\section{Necropsy}

At 24 weeks post-implantation, monkeys were deeply anesthetized with sodium Pentobarbital $(60 \mathrm{mg} / \mathrm{kg}$ i.m.) and Ketamine $(10 \mathrm{mg} / \mathrm{kg}$ i.m. $)$, and perfused transcardially with $0.9 \%$ saline $(500 \mathrm{ml}$ or more, until the draining fluid ran clear of blood). They were then infused with the fixative (4\% paraformaldehyde in $0.1 \mathrm{M}$ phosphate buffered saline) at $\mathrm{pH} 7.2$ (approximately 2.5 liters) via a catheter placed in the aorta. The brains were then removed and immersed in the fixative overnight at $4^{\circ} \mathrm{C}$. After rinsing with PBS, the brains were placed in 15\% sucrose/PBS for up to 5 days and then transferred to $30 \%$ sucrose/PBS solution and left at $4{ }^{\circ} \mathrm{C}$ until they had fully absorbed the sucrose solution and sunk to the bottom of the containers.

\section{Tissue preparation}

All tissue preparation was performed by an independent contractor, Dr. Jian Guan at the Liggins Institute, University of Auckland, New Zealand. Sequential coronal frozen sections $(50 \mu \mathrm{m})$ were cut on a sliding microtome (MICROM, HM450, MICROM international). Every 8th of these sequential sections was collected and pooled (e.g. sections 1, 9, 17, 25 and so on). The pools were numbered as pool 1 to pool 8 .

\section{Immunohistological staining and neuronal counting}

All immunohistochemical procedures were performed according to well-established standardized methods by an independent contractor, Dr. Jian Guan at the Liggins Institute, University of Auckland,
New Zealand. Briefly, sections were incubated with primary rabbit anti-human- $\mathrm{TH}$ (tyrosine hydroxylase) antibody (Protos Biotech Corporation, 1:1000) at $4{ }^{\circ} \mathrm{C}$ for 48 hours. After washing the primary antibody with PBS three times, sections were incubated with biotinylated goat anti-rabbit antibody (1:200, Sigma) at $4{ }^{\circ} \mathrm{C}$ overnight. After another round of washing with PBS, ExtrAvidin (Sigma, 1:200) was applied for 3 hours at room temperature, and then $0.05 \%$ 3,3-diaminobenzidine (DAB, InVitrogen) was added to visualize antibody-antigen complexes. Following labeling, the sections were mounted on glass slides and cover-slipped. For histological staining to assess potential tissue damage and cellular reaction, the sections were mounted on slides and stained with thionine (Baff Global) for 4 minutes. The stained sections were dehydrated, dried and cover-slipped.

\section{Quantification of TH immunoreactive fibers in the striatum}

Photographic images of $\mathrm{TH}$ staining were taken from both sides of the putamen $(1 \times)$ in all sections using a camera connected to Nikon800 microscope. The average density of TH staining and the background staining were photographed and the staining density was measured using ImageJ software (http://imagej. nih.gov/ij/). The staining density of TH was determined by subtracting the background staining. Both the total number of $\mathrm{TH}$ positive neurons and the number of TH neurons with dendrites and nuclei were counted manually for both sides of all sections analyzed.

\section{Post mortem histopathology analysis}

Hematoxylin and Eosin staining was performed on a 1-in-24 series of sections through the entire brain and evaluated by a Board Certified Veterinary Pathologist (Gribbles Veterinary New Zealand). Brain sections

Table 1

Neurological score rating

\begin{tabular}{lllll}
\hline Symptoms & Normal & Mildly abnormal & Abnormal/moderate & Severely abnormal \\
\cline { 2 - 5 } & 0 & 1 & 2 & 3 \\
\hline Posture & Normal & Mildly abnormal & Abnormal & Grossly abnormal \\
Balance & Normal & Impaired & Frequent falling & No movement \\
Tremor & Absent & Mild/not always & Moderate & Severe \\
Alertness & Normal & Reduced & Absent & \\
Motility at rest & Normal & Mild bradykinesia & Bradykinesia & Akinesia \\
Walking & Normal & Mildly reduced & Reduced & No walking \\
Motility, reaction to external stimuli & Normal & Mildly reduced & Reduced & Absent \\
Eyes & Normal & Reduced blinking & Eyes closed & \\
Head-checking movement & Present & Reduced & Absent & \\
\hline
\end{tabular}

Based on Takagi Y et al. (2005), Ref. 22. 
were also examined with thionine staining (see above). The neuropathology report indicates that only minimal or mild cellular reaction in brain tissue was seen in the three monkeys in the Sham group, one in the CP Treated group and one in the empty capsule group (data not shown).

\section{Microbiological analysis for potential pig virus transmission}

Post-mortem tissue samples (lung, spleen, liver, blood plasma and sera) were taken from two monkeys from the eCP Treated group and 2 monkeys from the Control/Sham group. DNA was isolated as described previously [24]. Total RNA was isolated from $25 \mathrm{mg}$ of liver, lung and spleen tissue and $200 \mu \mathrm{L}$ of plasma using the Nucleospin ${ }^{\circledR}$ RNA II Kit (MachereyNagel, Germany) according to the manufacturer's instructions. Each sample was spiked with $160 \mathrm{ng}$ of bacteriophage MS2 RNA (Roche Applied Systems, New Zealand) immediately prior to isolation. Total RNA was converted to cDNA using the Transcriptor High Fidelity cDNA Synthesis Kit and Random Hexamer Primers (Roche Applied Systems, NZ) according to the manufacturer's instructions. Real-time PCR for porcine endogenous retrovirus (PERV) was performed [24]. This multiplex assay is used to detect sequences from PERV pol, cytochrome oxidase subunit II (COII) and lambda bacteriophage in a single reaction. COII is included in order to detect microchimerism. PERV infection is only confirmed if it is detected in the absence of COII. Lambda is included as an internal control to confirm successful DNA extraction and to elucidate the effects of PCR inhibition if present.

\section{Statistics}

Behavioral responses and TH staining results were compared between groups by two-way ANOVA using Prism 5 (Graphpad Software Inc) with Bonferroni adjustment for multiple comparisons.

\section{RESULTS}

\section{Characterization of Encapsulated Porcine Chroid Plexus Cells (ePCs)}

The specialized cells in the choroid plexus are modified ependymal cells with epithelial cell characteristics. This single layer of cuboidal epithelial cells are supported by a layer of basal lamina underneath which is an extensive vascular bed containing fenestrated capil- laries, stromal cells, fibroblasts, immune cells as well as extensive neuronal fibres. These epithelial cells are connected to each other by tight junctions that effectively create a blood-cerebrospinal fluid (CSF) barrier. The apical surface of the epithelium contains many villi that generate active movement of fluid and secreted factors. The primary function of the $\mathrm{CP}$ is to produce $\mathrm{CSF}$, a process that can occur at a rate of $500 \mathrm{mls} /$ day in humans along with many bioactive components including growth factors, neurotrophins, anti-oxidants, and protein chaperons [1-5].

$\mathrm{CP}$ clusters used in our experiments are prepared by an enzymatic and mechanical digestion of harvested neonatal porcine choroid plexus followed by several washing steps to produce clusters of an average size of $70 \mu \mathrm{m}$ in diameter. Immunohistological analysis using an antibody to tight junction protein ZO-1 reveals that greater than $99 \%$ of the CP clusters contained ZO-1positive epithelial cells (Fig. 1A). A flow cytometric analysis of single cell preparation indicates that our $\mathrm{CP}$ preparation consists of greater than $80 \% \mathrm{ZO}-1$ positive epithelial cells at the time of encapsulation (Fig. 1B). The remaining population includes vascular endothelial cells (SMA+) and fibroblasts (Vimentin+), but there were no neurons (NeuF-) or astrocytes (GFAP-) (data not shown). Encapsulation with alginate is carried out as previously described [6-10], and eCPs are visually inspected and the $\mathrm{CP}$ viability is confirmed using Calcein-AM prior to transplantation (Fig. 1C, D).

\section{Establishment of PD model using MPTP}

DA lesions were induced in Rhesus monkeys (Macaca mulatta) by a well-established method using MPTP, a precursor of the nigrostriatal mitochondrial poison MPP+, which has long been recognized for its ability to degenerate DA neurons in the $\mathrm{SN}$ and induce Parkinsonian symptoms in humans and nonhuman primates [15, 16, 19-23, 25-31]. A schematic representation of our experimental design is illustrated in Fig. 2A. Male Rhesus monkeys of average age of 8 years and of average body weight $(8.5 \mathrm{~kg})$ were used in the experiment (see Suppl. Material S1 for age and weight of each animal). Prior to administering MPTP, the monkeys were assessed for pre-lesion behavioural analysis and deemed to be normal in all neurological categories used in our analysis (Table 1). To induce acute intoxication, $1.5 \mathrm{mg} / \mathrm{kg}$ of MPTP was infused into internal carotid artery at a rate of $2 \mathrm{ml} /$ minute with the guidance of angiography. The dose of MPTP was chosen based on other non-primate studies using direct injection of the drug into carotid 

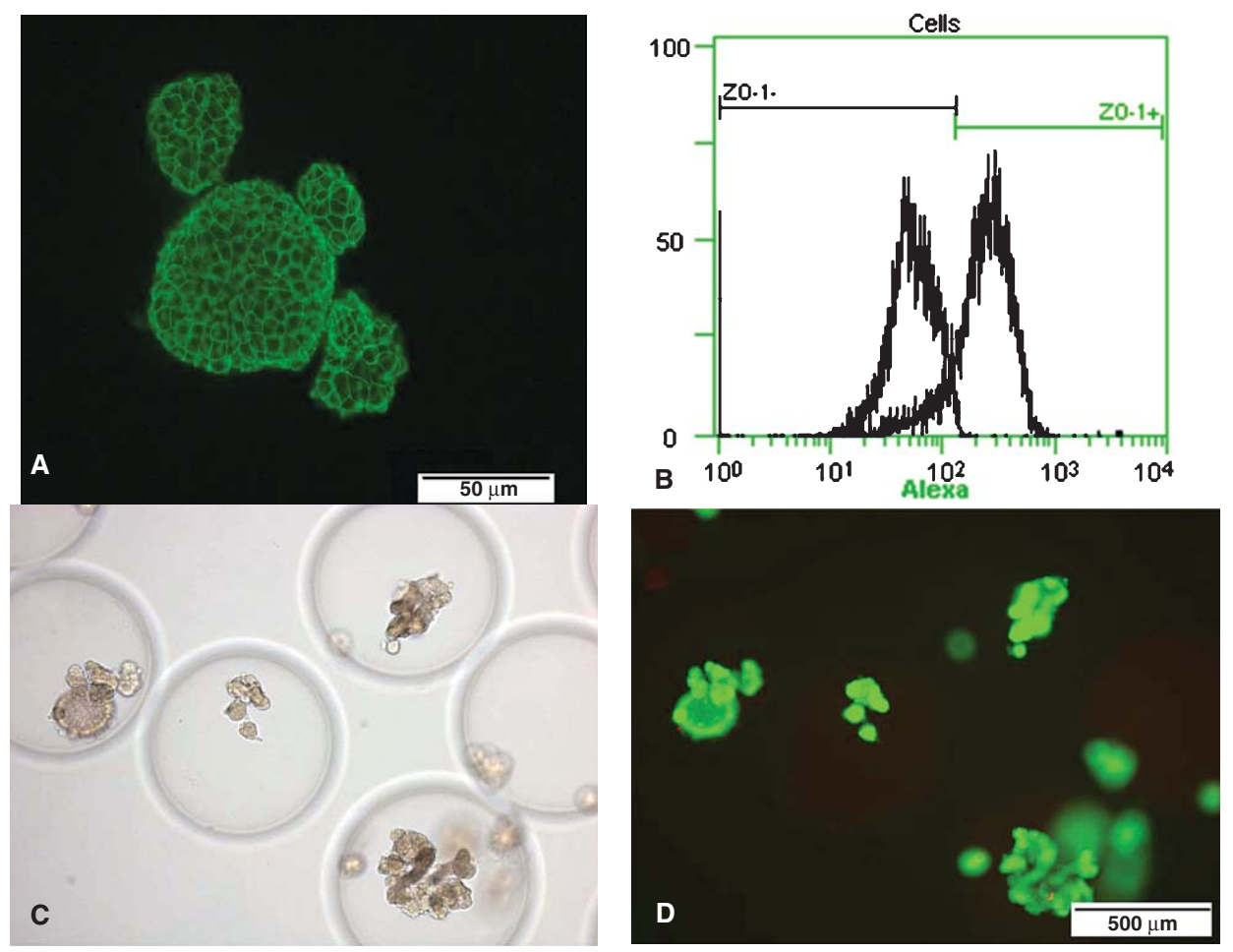

Fig. 1. The purity and viability of neonatal pig choroid plexus cell clusters. The purity of clusters was assessed by the presence of tight junction ZO-1 positive cells at day 7 in culture. (A) Free clusters of CP stained for tight junction with anti-ZO-1 antibody (in green); (B) Analysis by flowcytometry with anti-ZO-1 antibody indicating that approximately $80 \%$ of cells express $\mathrm{ZO}-1$ at the time of encapsulation. An isotype secondary antibody control separates to the left as ZO-1-negative; (C) Encapsulated CP clusters under bright field microscope (average diameter of each capsule is about $600 \mu \mathrm{m}$ ); (D) Viability of clusters at the time of transplantation as assessed by Calcein-AM with minimal Ethidium Bromide staining.

artery to induce acute and stable DA lesion of the nigrostriatal pathway [19-21]. Within four (4) weeks after the MPTP administration, all of the monkeys displayed neurological impairment in the following categories: alertness, head-checking movement, eye movement, posture, balance, motility at rest, reaction to external stimuli, walking, and tremor. To determine the extent of damage specific to DA neurons, we employed an apomorphine challenge which has been used to elicit circling behaviour when an imbalance in dopamine activities between the two hemispheres of the brain is exacerbated by MPTP treatment [23]. While the circling behaviour itself is not directly relevant to PD and each animal's response varies, the changes in the circling score in the same animal in a longitudinal study can be used as readout for either improvement or further deterioration of the DA neural pathway. Circling of more than 7 asymmetrical turns per minute upon apomorphine challenge was considered to be a sign of adequate lesion. The monkeys were observed for an additional four weeks (total of
8 weeks post-MPTP treatment) to rule out any spontaneous recovery that is known to occur in MPTP-treated monkeys over this period post-MPTP treatment [16, 28-30]. We observed spontaneous neurological recovery in two monkeys, and they were excluded from further study. The monkeys with stable lesions were randomized into three groups: i) Sham group (S, $n=3)$; ii) empty capsule-only implanted Control group (Control, $n=6$ ); and neonatal porcine eCP Treated group (Treated, $n=6$ ) (Fig. 3A, Pre-Tx). The grouping was done to minimize the difference in the number of turns per hour at baseline between the 3 groups (Treated group $913 \pm \mathrm{SE} \mathrm{171;} \mathrm{Control} \mathrm{group} 624 \pm 145$; and Sham group $838 \pm 366$ turns per hour) (Fig. 4A, Pre-Tx).

\section{Improvement in neurological scores in monkeys that received neonatal porcine eCP implants}

Between 8 to 12 weeks after the administration of MPTP, surgeries were performed to place sham surgery 
A

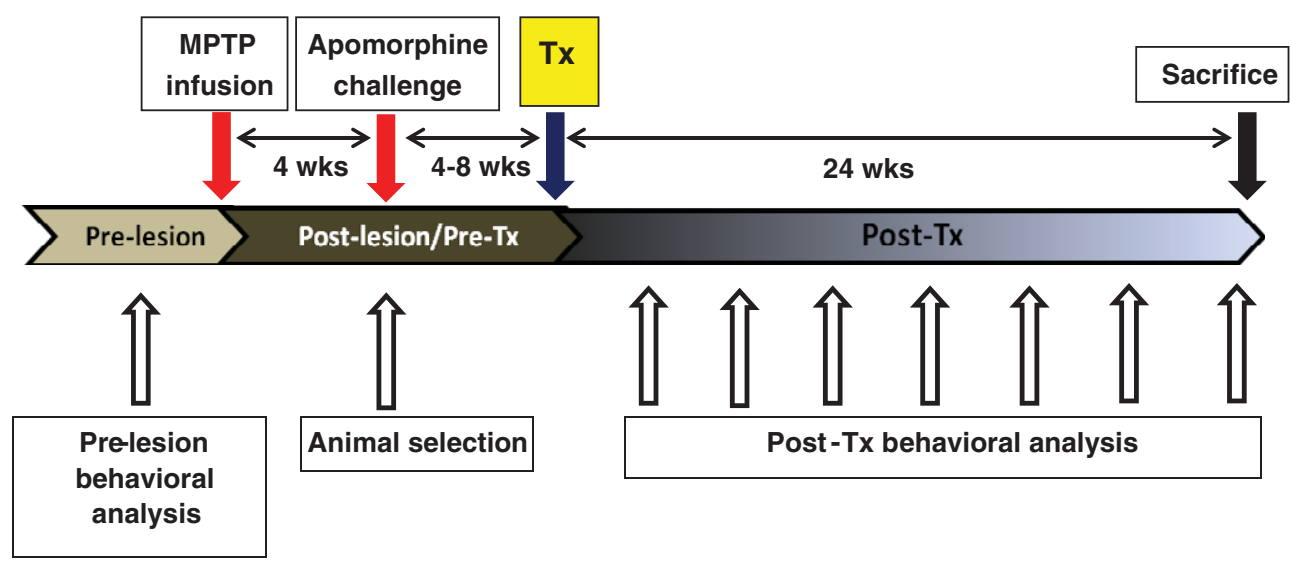

B

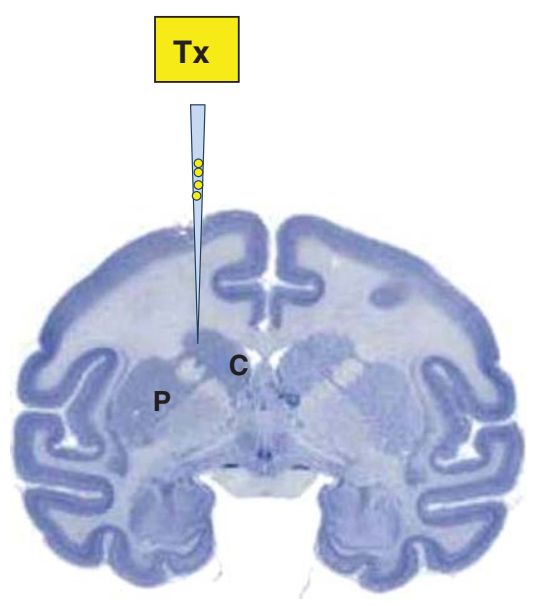

Fig. 2. (A) A schematic diagram of the study design; (B) Illustration of the implant site on the striatum; transplant (Tx), caudate nucleus (c), putamen (p) (brain image from Brainmaps.org).

(Sham or $\mathbf{S}, n=3$ ), empty capsule implants (Control or $\mathbf{C}, n=6$ ) or eCPs implants (Treated or $\mathbf{T}, n=6$ ) into the right caudate nucleus using $\mathrm{CT}$ imaging (Fig. 2B). Each animal in the Control and Treated groups were implanted with 40 empty capsules and capsules containing approximately $2 \times 10^{4} \mathrm{CP}$ cells, respectively. Using a behavioural rating scale by Takagi et al. [22] (Table 1), the neurological scores were measured at $2,4,8,12,16$ and 24 weeks following the surgical procedure by observers blind to the types of treatment received by each animal. While the neurological scores changed little in the Sham and Control groups, a clear improvement was evident within four weeks after implantation in all Treated animals that received neonatal porcine eCP implants (Fig. 3). Among the animals in the Treated group, two patterns of response emerged. Four animals, T1, T2, T3, and T4, continued to show improvements in neurological scores for the entire 24 weeks, resulting in approximately $48 \%, 70 \%, 47 \%$, and $31 \%$ improvements at 24 weeks, respectively (Fig. 3A, B, D). However, two remaining animals, T5 and T6, showed improvements in neurological scores for the first 12 and 8 weeks, respectively, but regressed during the remaining months, resulting in approximately $22.7 \%$ total improvement at 24 weeks (See Suppl. Material S2). Thus, the ranking order of the monkeys that displayed most improvements in neurological scores was: $\mathrm{T} 2>\mathrm{T} 1>\mathrm{T} 3>\mathrm{T} 4>\mathrm{T} 5=\mathrm{T} 6$ (Fig. 3A, B, D). At 24 weeks, the average improvement in neurological scores in the $\mathrm{T}$ group $(39.2 \%)$ 

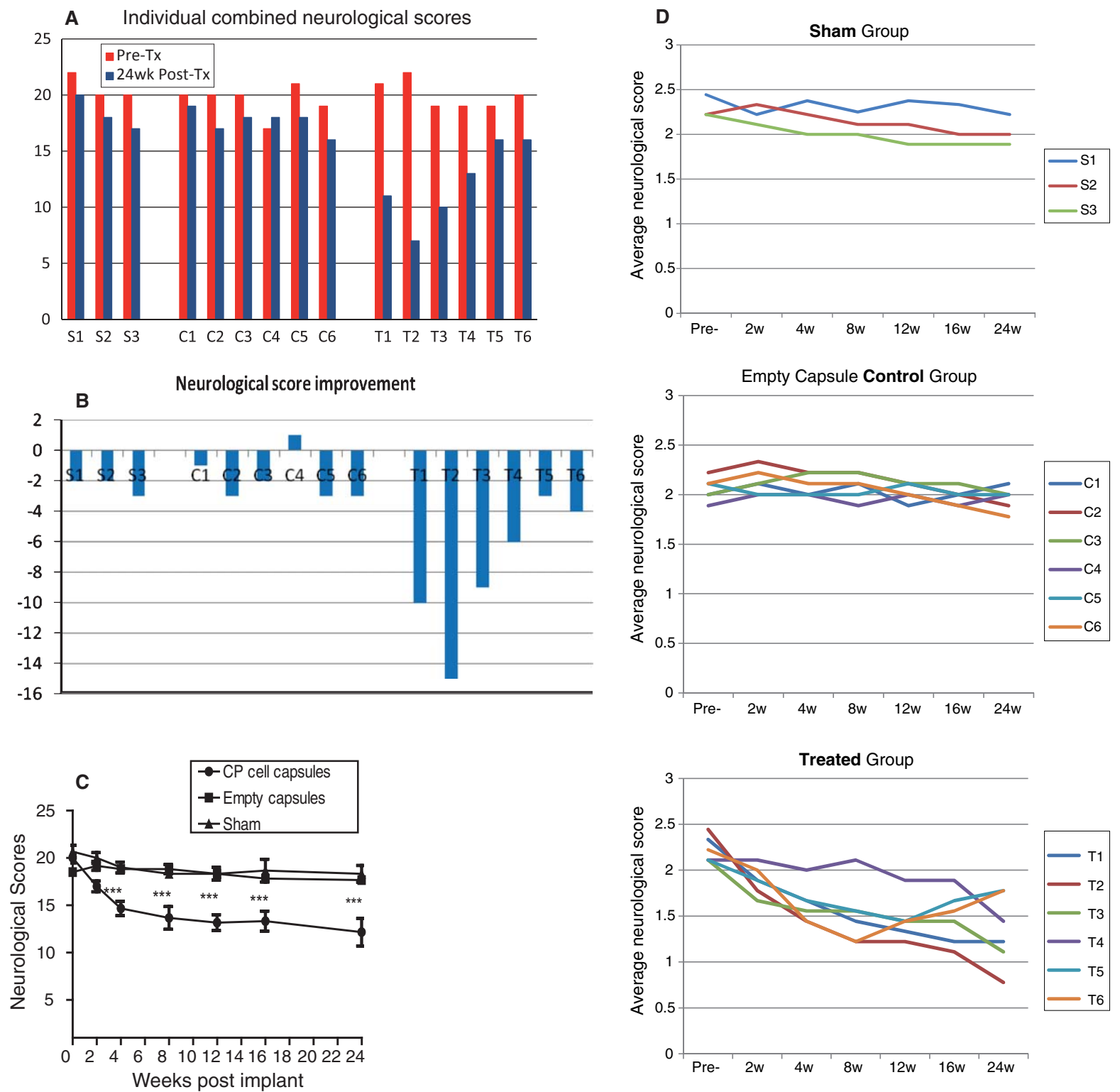

Fig. 3. (A) Individual combined neurological score assessment at 4 weeks after MPTP-induced lesion (in red) and at 24 weeks after transplantation (Tx) (in blue); sham (S), empty capsule control (C) and eCP treated (T); (B) Individual combined neurological score improvement achieved between 4 week post-lesion and 24 week post-Tx; (C) A chronological representation of the average combined neurological scores of each group; (D) A chronological representation of combined neurological scores of individual monkeys; (E) The average group neurological score improvement in each symptom category.

was significantly different from that of $\mathrm{C}(4.9 \%)$ or $\mathrm{S}$ $(10.9 \%)$ groups $(p<0.001)$ (Fig. 3C).

We next examined which of the neurological categories were most affected by implantation of eCPs. When we compared the average group improvements within each category, the degree of improvement was not equal in all categories. Compared to controls, the Treated group showed statistically significant recovery $(p<0.05)$ in posture, balance, tremor, and alertness (Fig. 3E). Amongst the neurological scores that improved both motility at rest and the walking score were not statistically significant. eCP treatment did not result in any measurable recovery in motility (reaction to external stimuli), eye movements, and head checking movements compared to the control groups. 
E

Posture

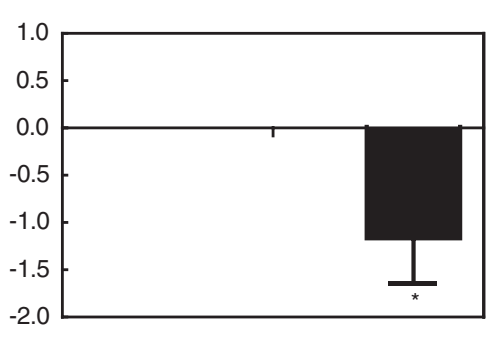

Alertness

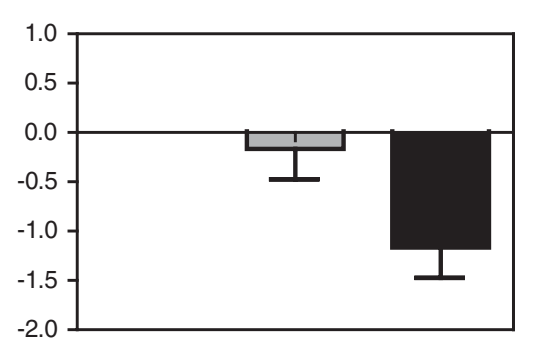

Motility, reaction to external stimuli

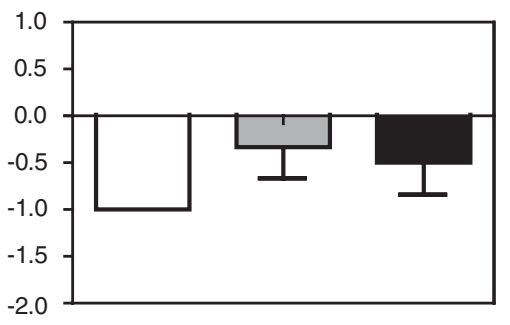

Sham
Balance

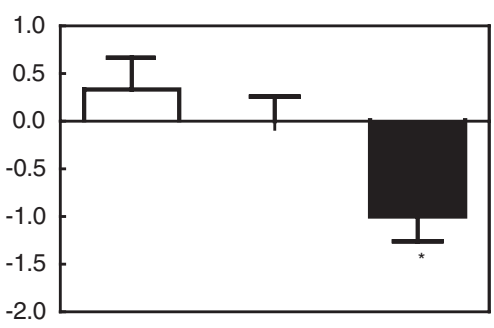

Walking

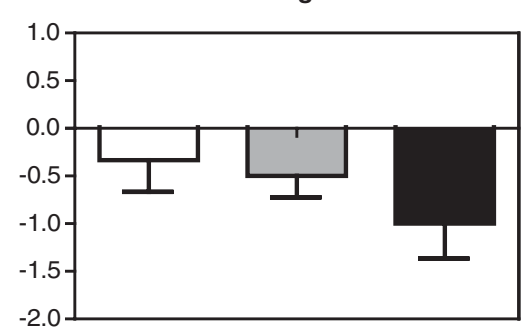

Eyes

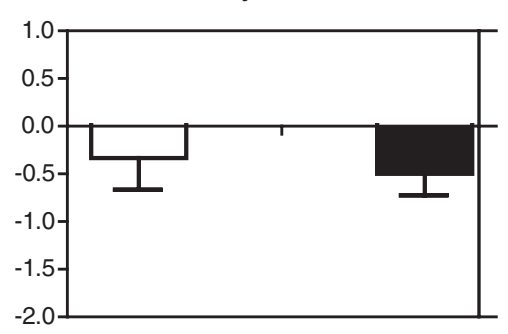

Control
Tremor

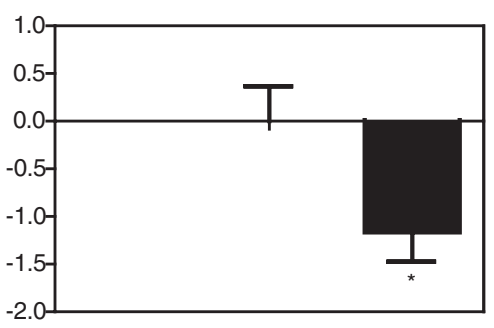

Motility, at rest

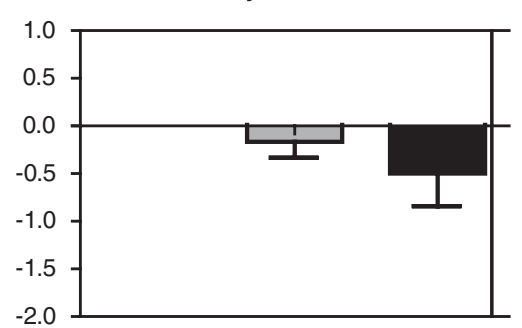

Head-checking movement

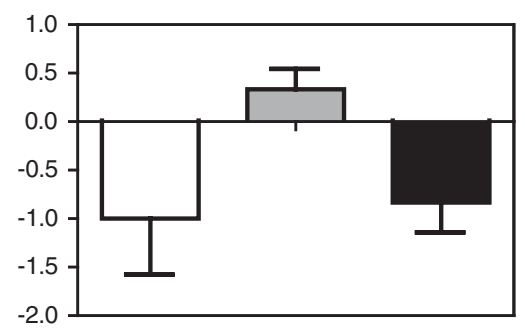

Treated

Fig. 3. (Continued)

Table 2

Scores reflecting neurological function

\begin{tabular}{lccc}
\hline & \multicolumn{2}{c}{$\%$ Reduction of group total score after 24 weeks } \\
\cline { 2 - 4 } & CP capsules $N=6$ & Empty capsules $N=6$ & Sham $N=3$ \\
\hline Posture & 50.0 & 0 & 0 \\
Balance & 42.9 & 0 & {$[16.6]^{*}$} \\
Tremor & 41.2 & 0 & 0 \\
Alertness & 63.6 & 9.1 & 0 \\
Walking & 37.5 & 20.0 & 12.5 \\
Motility, at rest & 25.0 & 7.8 & 0 \\
Motility, to external stimuli & 21.4 & 15.3 & 33.3 \\
Eyes & 27.3 & 0 & 16.6 \\
Head checking movement & 45.4 & 20.0 & 42.9 \\
\hline
\end{tabular}

The difference in the total scores at week 0 baseline and 24 weeks after capsule or sham implant for each group is expressed as \% reduction to reflect improvement in function. *[\% increase]. 

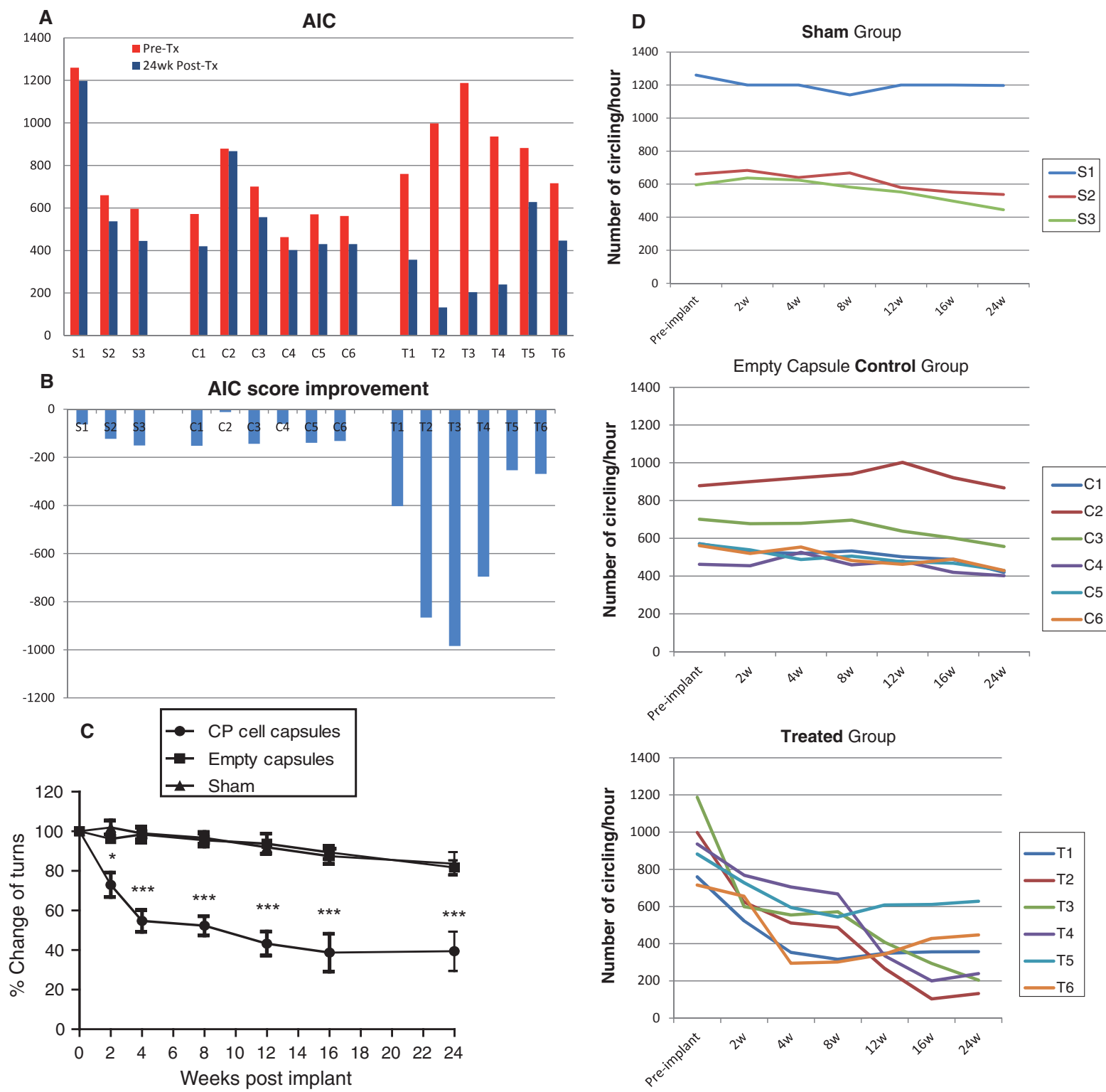

Fig. 4. (A) Apomorphine-induced circling (AIC) score assessment at pre-Tx (in red) and at 24 weeks after transplantation (in blue); (B) Individual AIC score improvement achieved between pre-Tx and 24 weeks post-Tx; (C) A chronological representation of the average combined AIC scores of each group; (D) A chronological representation of combined neurological scores of individual monkeys.

\section{Improvement in circling scores after apomorphine challenge}

When observed without apomorphine stimulation, the monkeys implanted with eCPs were more alert, displayed better posture and balance, and had fewer tremors compared to those measures of the Sham and Control groups. The apomorphine-induced circling (AIC) score was recorded at pre-implantation stage and at 2, 4, 8, 12, 16, and 24 weeks after the implant surgeries by observers blind to the treatment received by each monkey. As shown in Fig. 4A and $4 \mathrm{D}$, a large variation in the AIC score was observed at pre-implantation stage of MPTP-treated monkeys, a phenomenon also reported by others [23]. Despite such individual variations, each monkey in the Sham and Control groups displayed consistently similar AIC scores during the entire 24 weeks, indicating that the AIC score reflects a stable state of deficits in the DA pathway (Fig. 4C, D). As was the case in the neurolog- 
ical study, we observed a significant improvement in AIC scores only in the Treated group. T2, T3, and T4 made significant and continuous improvements in AIC score for the entire 24 weeks. T1, which showed continuous improvement in the neurological score, however, displayed a stabilized AIC score around eight weeks post-surgery. In a manner parallel to the neurological study, T5 and T6 showed significant improvements in AIC scores until week 8 and week 4, respectively. However, they regressed during the remaining period until week 24, but still achieved overall improvement in AIC scores at the end of 24 weeks. At 24 weeks after the implantation, the reduction in AIC score was $60.6 \%$, $19.2 \%$ and $16.4 \%$ for the Treated, Control and Sham groups respectively $(p<0.001)$. Interestingly, the time course of AIC recovery appears to be predictive of the pattern of neurological recovery (see Suppl. Material S2). Such correlation between neurological score and AIC improvements, not only in the degree, but also in the trend over time, strongly supports the ability of eCPs to restore neurological function in a non-human primate model of Parkinson's Disease.

\section{Immunohistological examination of $\mathrm{TH}+$ neurons in the post-mortem brains}

To determine the extent of MPTP-induced damage to the nigrostriatal region of the monkey brains, we first measured the number of tyrosine hydroxylaseimmunoreactive $(\mathrm{TH}+)$ DA neurons in in the substantia nigra (SN) of each monkey at the completion of the experiment. Eight $50 \mu \mathrm{m}$ thick representative sections from the nigrostriatal region of each brain were collected and stained for TH. The total number of $\mathrm{TH}+$ neurons from each set is shown in Fig. 5 (The brain of T1 was severely damaged during transport and processing, and the SN could not be seen in the brain section of S1, and therefore we had to exclude any neuronal counts collected during histological analysis of these animals). Quantitative assessment of the number of $\mathrm{TH}+$ neurons in the $\mathrm{SN}$ in each group twenty four (24) weeks after surgical implants showed that there was a significantly high $(p<0.05)$ number of $\mathrm{TH}+$ neurons in the $\mathrm{SN}$ of the Treated group compared to those of the Control and Sham groups (Fig. 5B).

When analysing individual monkeys, however, a direct correlation could not be made between the total number of $\mathrm{TH}+$ neurons in the $\mathrm{SN}$ and the neurological score improvements. All of the animals in the T group had relatively higher numbers of $\mathrm{TH}+$ neurons compared to the sham group. T2 and T3, which displayed best improvement in neurological scores along with $\mathrm{T} 1$, had the highest number of $\mathrm{TH}+$ neurons in the $\mathrm{SN}$. Interestingly, T2 and T3 had more $\mathrm{TH}+$ neurons on both sides of the brain compared to other animals, suggesting a bilateral rescue effect. Surprisingly, two animals in the $\mathrm{C}$ group, $\mathrm{C} 2$ and $\mathrm{C} 3$, had a large number of $\mathrm{TH}+$ neurons compared to the rest of the control animals. C3 had a relatively high number of $\mathrm{TH}+$ neurons on the left side while $\mathrm{C} 2 \mathrm{had}$ a large number of $\mathrm{TH}+$ neurons on the right side of the brain. Despite the presence of a substantial number of $\mathrm{TH}+$ neurons in the $\mathrm{SN}$, however, neither $\mathrm{C} 2$ nor $\mathrm{C} 3$ displayed any discernible improvement in neurological or AIC scores, indicating that neuronal numbers alone could not account for the neurological improvement observed in our study.

In summary, although there was some correlation with the number of $\mathrm{TH}+$ neurons and improvements in the neurological score (e.g., T2, which had the most neurological improvement had the most number of $\mathrm{TH}+$ neurons), we could not find a direct correlation between these neuronal counts alone and neurological improvements in all animals. Based on these results, we believe that increase in neuronal number is necessary, but not sufficient to affect behavioural recovery.

\section{TH staining intensity and DA neuronal innervation in the striatum}

Because the high $\mathrm{TH}+$ neuronal count in the control C2 did not correlate with an expected behavioural recovery, we performed additional comparative analysis of sections from $\mathrm{C} 2$ and $\mathrm{T} 3$ that had comparable number of SN neurons. In an effort to find histological evidence to explain this discrepancy, we investigated the extent of DA innervation into the SM territory of the striatum by staining the midbrain sections of $\mathrm{C} 2$ and $\mathrm{T} 3$ with antibodies to $\mathrm{TH}$ (Fig. 6A, B). The TH staining density of sections from $\mathrm{T} 3$ was four times higher than that from $\mathrm{C} 2$ (Fig. 6C). Higher magnification image analysis revealed that many $\mathrm{TH}+$ striatal fibres were associated with the striatum in T3, but not in $\mathrm{C} 2$ (Fig. 7A).

In addition to finding higher $\mathrm{TH}+$ staining density in the SM territory, the associative territory of the precommissural region was analysed. The study revealed a striking difference between the T3 and C2; a dense network of $\mathrm{TH}+$ fibres was present in the right striatum of T3 but not in $\mathrm{C} 2$ (Fig. 7B). A dense fibre network in the implanted striatum was found only in the Treated group in a ranking order that matches the neurological outcome i.e., most dense in T2, T3 and less so in $\mathrm{T} 4$ but not in the striatum of $\mathrm{T} 5$ and $\mathrm{T} 6$ both of which 


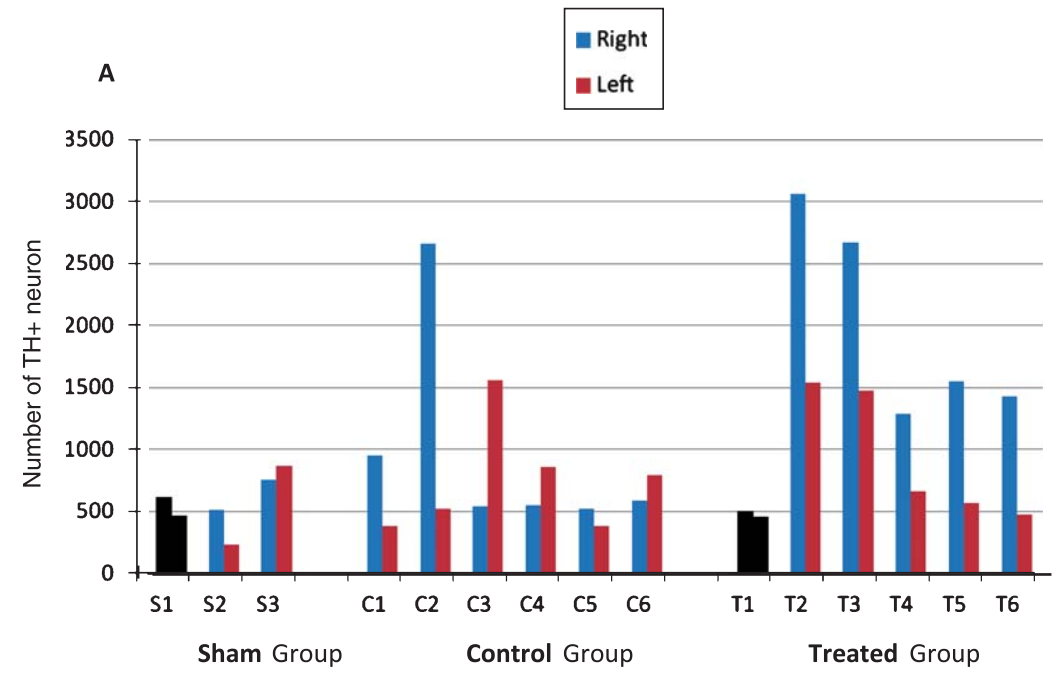

B

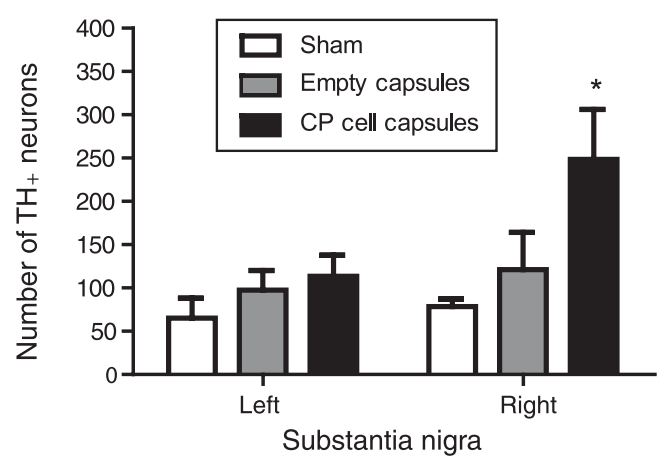

Fig. 5. (A) The number of tyrosine hydroxylase-immunoreactive (TH+) neurons in the substantia nigra. Total counts of TH+ neurons from eight $50 \mu \mathrm{m}$ serial sections of the right (in blue) and left (in red) mid-brain region. The sections of T1 and S1 were damaged or not seen and their neuronal counts (in black) could not be used for neuronal count assessment; (B) The average number of tyrosine hydroxylase-ir (TH+) neurons per group $(p<0.05)$.

displayed regression in neurological scores and AIC scores. These observations suggest that the improvement of neurological function in the Treated group may be mediated by the ability of eCPs to restore $\mathrm{TH}+$ fibres in the striatum.

\section{Analysis of the post-mortem tissues}

We have previously reported retrieval of implanted eCP capsules from our rat study to show that CP cells were viable for at least six months in vivo [6]. Recovery and characterization of these capsules of approximate diameter of 600-660 $\mu \mathrm{m}$ in a total injection volume of $20 \mu 124$ weeks after implantation required destruction of the brain tissues in these earlier studies, a process we could not afford to perform in the current primate study. The frozen monkey brains were sec- tioned in $50 \mu \mathrm{m}$ thickness on a sliding microtome and washed multiple times during the process. An independent pathology report from the Gribbles Veterinary Group confirms that there were no clear catheter tracks found in any sections stained with thionine or haematoxylin and eosin staining, and our search to find any intact CP clusters within the rest of tissue sections was unsuccessful. The alginate capsules were damaged by histological processing but artefactual remnants were visible in the striatum of four monkeys adjacent to the internal capsule.

To assess host response to eCP implantation, we performed immunohisotochemical analysis using antibodies to the astrocytic protein Glial Fibrillary Acidic Protein (GFAP) and to isolectin B4 (IB4), a commonly used microglial marker in the brain [32]. Examination of the brain sections stained with GFAP and IB4 
Treated (T3) Control (C2)

A

Sensorimotor

(Striatum)

B

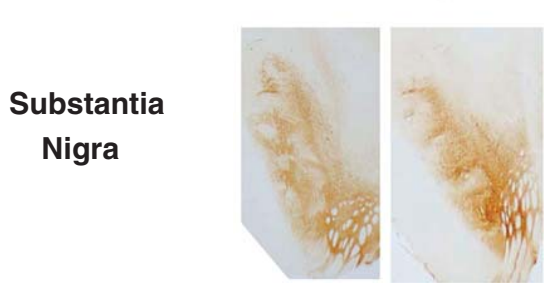

C

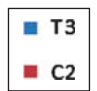

TH density of putamen and caudate nuclues

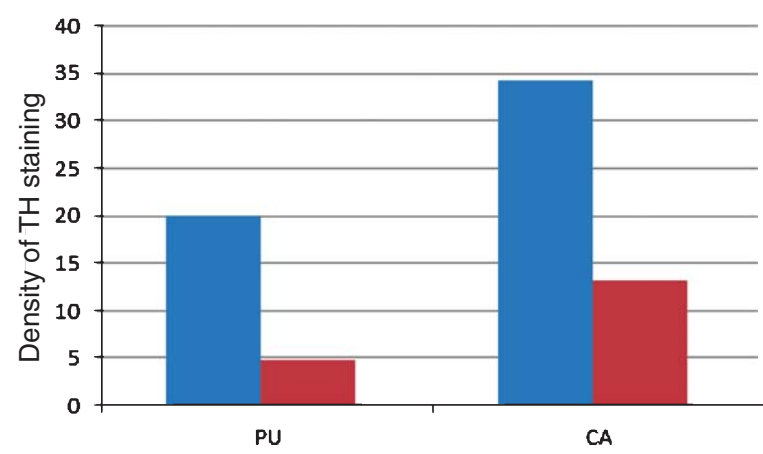

Fig. 6. TH staining of the striatum and SN. The TH staining in the sensorimotor (SM) territory of the striatum is strong in the treated monkey with good neurological improvement (T3) but not in a control monkey without neurological improvement (C2). The SN of both animals had comparable number of neurons.

indicate that no evidence of increased astrogliosis or microgliosis is found in the Treated compared to the Sham group (see Suppl. Material S3). As expected, staining of ramified microglia was detected in S3 outside the striatum, but no IB4 stained microglia was visible in the striatum of all three groups. Antibody to IB4 is known to stain blood vessels which we were able to detect in all sections. With no demonstrable discrete cell inflammatory response, there is a possibility that the tissue reactions to the surgical intervention and the capsule implantation had been reabsorbed during the six month interval between implantation and necropsy. The pathology report confirms that there was no evi- dence of inflammation or edema of the brain tissues of these monkeys.

\section{DISCUSSION}

Two different therapeutic approaches can be taken to address the neuronal and striatal deficits in the PD brain: i) supplying exogenous cells to replace degenerated DA; or ii) protecting striatal degeneration of DA fibres using targeted introduction of vehicles that secrete neural protective factors. We have taken the second approach of neuroprotection using eCP clusters to develop therapies for PD. Our approach is based on the demonstrated ability of eCPs to prevent neural degeneration and to restore neural functions [6-10].

The MPTP-treatment is well-established and the most relevant method to generate PD symptoms in monkeys, including resting tremor, a symptom that is difficult to generate in other animal models [31]. However, this method has some limitations: individual animal response to MPTP is quite variable and spontaneous recovery has been known to occur frequently within 3 to 5 weeks after MPTP treatment [28-30]. Indeed, two of the monkeys in our study recovered spontaneously within 4 weeks of the administration of MPTP and were excluded from further study. All of the remaining monkeys displayed a stable lesion until eight weeks after MPTP treatment at which time the eCP implantation studies were initiated. It is noteworthy that all of the monkeys in both control groups displayed stable neurological deficits and AIC scores throughout the entire duration of this study ( $>6$ months).

Significant improvements in neurological and AIC scores were only observed in animals that received eCP implantation. While regression in these scores was observed in two animals starting 4-8 weeks post-transplantation, the other four animals (T1-T4) continued to show improvements for the entire duration of the study which lasted 24 weeks. In a longitudinal study, the AIC score appears to be predictive of neurological state of the animal: the change in AIC score preceded the changes in neurological scores by about two weeks. However, the two scores did not match identically, suggesting that improvement in other neural pathways may be reflected in the neurological score improvements.

In animals implanted with eCPs, a statistically significant improvement was observed in posture, balance, tremor, and alertness all of which are important aspects of PD treatment. Although the improvement in walking by the Treated group in our study was not 
A

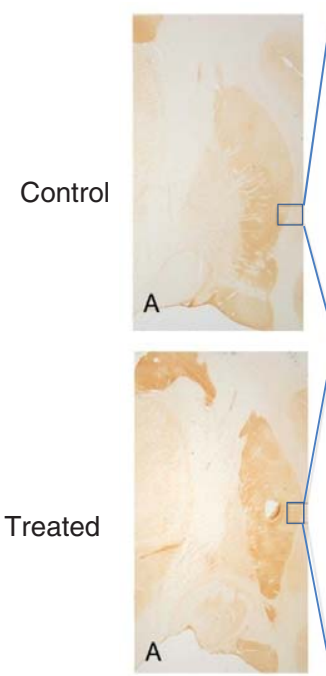

B

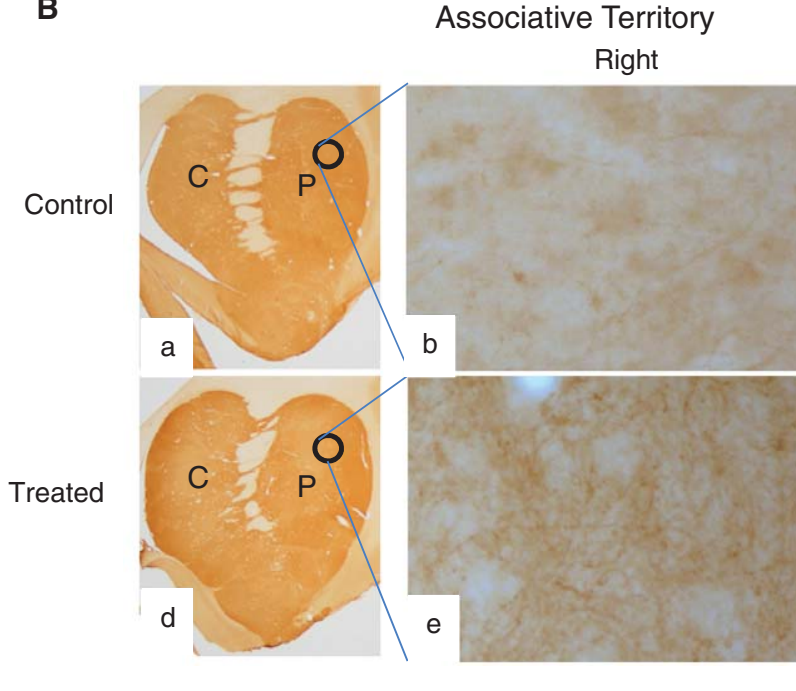

Sensorimotor Territory
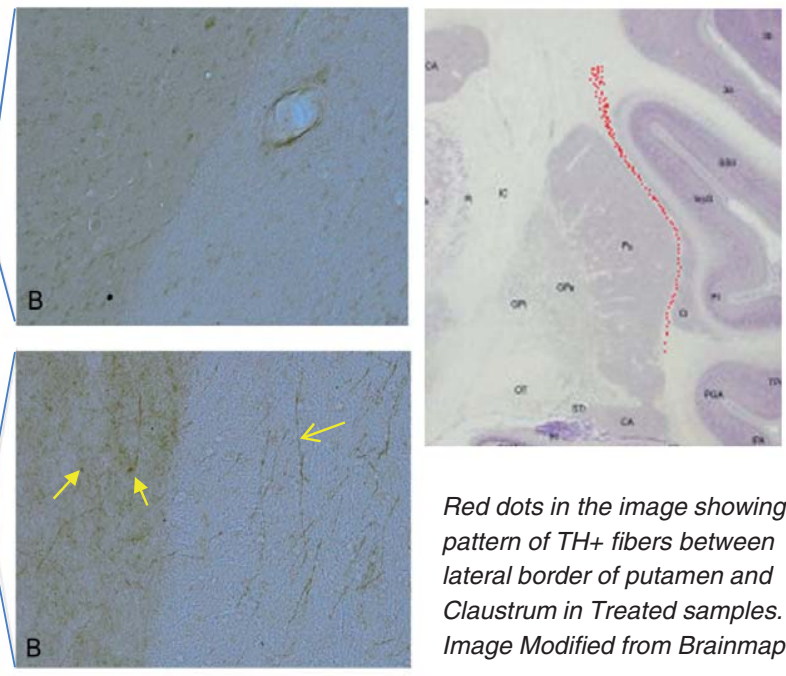

Red dots in the image showing the pattern of $\mathrm{TH}+$ fibers between lateral border of putamen and Claustrum in Treated samples. Image Modified from Brainmaps.org

Fig. 7. TH-fibre staining in the striatum. (A) TH fibre staining in the sensorimotor (SM) striatum (post-commissural); (B) TH fibre staining in the associative region of the striatum (pre-commissural); A dense network of TH+ neural fibres are visible in the Treated, but not in the Control striatum.

statistically significant, it is one of the symptoms that improved in the Treated animals overall $(37.5 \%)$ and in $\mathrm{T} 2$ in particular $(66 \%)$. It is also the case that walking score measurement in humans, such as the timed 'up \& go' test as well as Gait analysis by Movement Disorder Society-Unified Parkinson's Disease Rating Scale (MDS-UPDRS), involves walking a specific distance (10 meters) under instruction, whereas in animals, only spontaneous walking is measured [33].

The cellular basis of behavioural improvement was investigated by post-mortem analysis of DA neurons of SN. Even though MPTP was injected into the right carotid artery to favour unilateral lesion, the majority of the Sham and Control groups had a small number of $\mathrm{TH}+\mathrm{DA}$ neurons on both sides of the brain, indicating that the MPTP treatment had bilateral effects in the majority of these monkeys. We believe that the bilateral lesion may have resulted from a relatively high dose of MPTP used in our study $(1.5 \mathrm{mg} / \mathrm{kg})$ [19] in addition to the fact that the ligation of external carotid artery $[20,21]$ was not performed in the current study.

The total number of DA neurons in the SN did not strictly correlate with the behavioural improvement, although the monkeys with the most behavioural 
improvements (e.g., T2 and T3) had in general more $\mathrm{TH}+$ neurons on both sides of the brain compared to those that displayed moderate improvements (i.e., T4, $\mathrm{T} 5$ and T6). We were surprised to find that the two animals that received only empty capsules, $\mathrm{C} 2$ and $\mathrm{C} 3$, had a relatively large number of $\mathrm{TH}+$ neurons in their respective $\mathrm{SN}$, but displayed no significant improvement in either the behavioural or AIC scores. We investigated possible reasons for this discrepancy and found that they had little, if any, TH+ neural fibres in their SM and associative territories of the striatum. A deficit in motor function accompanied by striatal depletion of DA terminals without nigral cell loss has been observed in a MPTP-treated animal model of PD by Willis and Donnan [34]. Because C2 and C3 did not display any improvement in their neurological or AIC scores, it is unlikely that these neurons represent spontaneously recovered functional DA neurons. We concluded from these results that i) a significant number of $\mathrm{TH}+$ neurons in the substantia nigra is necessary, but not sufficient on its own to result in behavioural recovery; and ii) the extent of striatal fibre density is the key determinant in the behavioural outcome.

Several possible striatal dopaminergic compensation mechanisms have been proposed to explain the striatal recovery without increasing the number of DA neurons: i) enhanced dopamine secretion by remaining fibres [35-37]; ii) increase in the extracellular level of DA or decrease in DA uptake [37-41]; iii) sprouting of collateral fibres from the remaining fibre terminals in the limbic territory of the striatum or nearby pallidum $[42,43]$; iv) increased sensitivity to dopamine by the striatal neurons [44]; v) de novo production of DA by neurons intrinsic to the striatum $[34,45,46]$. The data presented here suggest that implanted porcine eCPs promote more $\mathrm{TH}+$ fibres in the associative region of the pre-commissural striatum and increased production of $\mathrm{TH}$ in the lesioned non-human primate striatum (Fig. 7).

In summary, we have demonstrated that implanting eCPs, known to secrete many neuroprotective and neurorestorative factors, into the affected site of the primate model of PD has resulted in improvements in neurological behaviours along with the restoration of striatal neural fibre networks. This result is consistent with our previous work demonstrating that porcine eCPs display neurorestorative function in various animal models of neurodegenerative diseases. Remarkably, such lasting therapeutic effects in monkey brains were observed with only 40 capsules containing eCPs. Taken together, our study support the potential utility of porcine eCPs in treatment of Parkinson's Disease.

\section{ACKNOWLEDGMENTS}

We are grateful to the following individuals/groups without whose involvement this work would not have been possible. H Lin and XM Luo, first co-authors, were equally responsible for executing and coordinating this study. Lei Luo and Qiong Dong of The Third Xianya Hospital of Central-South University, China provided experimental and surgical support; Professor Bin Qiu of The Second Xiangya Hospital of Central-South University, China assisted with locating co-ordinates for stereoscopic surgery; Zhiyong $\mathrm{Xu}$ and Peng Cai of Gaoyao Kangda Laboratory Animal Science \& Technology Co Ltd, China were responsible for animal care and surgical support; all striatonigral tissue harvest, sectioning, immunohistological staining, and neuronal counts in the $\mathrm{SN}$ were provided by Jian Guan and her staff at the Liggins Institute, University of Auckland, New Zealand, on a contractual basis; staff at Living Cell Technologies provided animals and tissues from designated pathogen-free pig breeding facility, as well as surgical and production help; Dr Keith McSporran of Gribbles Veterinary New Zealand, provided histopathology services; Dr. Edward Saravolac at LCT provided critical reading of the manuscript and insightful comments.

\section{CONFLICT OF INTEREST}

Hai Lin, Marilyn S Geaney, Lee Law, Shaun Wynyard, Shamim B Shaikh, Robert B Elliott, Jacqueline E Lee, and Paul L-J Tan are employees of Living Cell Technologies.

\section{SUPPLEMENTARY MATERIAL}

The supplementary figure is available in the electronic version of this article: http://dx.doi.org/10.3233/ JPD-130214

\section{REFERENCES}

[1] Redzic ZB, Preston JE, Duncan JA, Chodobski A, \& Szmydynger-Chodobska J (2005) The choroid plexuscerebrospinal fluid system: From development to aging. Curr Top Dev Biol, 71, 1-52.

[2] Chodobski A, \& Szmydynger-Chodobska J (2001) Choroid Plexus: Target for polypeptides and site of their synthesis. Microsc Res Tech, 52(1), 65-82.

[3] Ghersi-Egea JF, \& Strazielle N (2001) Brain drug delivery, drug metabolism, and multidrug resistance at the choroid plexus. Microsc Res Tech, 52(1), 83-88.

[4] Emerich DF, Vasconcellos A, Elliott RB, Skinner SJM, \& Borlongan CV (2004) The choroid plexus: Function, pathology 
and therapeutic potential of its transplantation. Expert Opin Biol Ther, 4(8), 1191-1201.

[5] Emerich DF, Skinner SJ, Borlongan CV, Vasconcellos AV, \& Thanos CG (2005) The choroid plexus in the rise, fall and repair of the brain. Bioessays, 27(3), 262-274.

[6] Skinner SJ, Geaney MS, Lin H, Muzina M, Anal AK, Elliott RB, \& Tan PLJ (2009) Encapsulated living choroid plexus cells: Potential long-term treatments for central nervous system disease and trauma. J Neural Eng, 6(6), 65001.

[7] Borlongan CV, Skinner SJ, Geaney M, Vasconcellos AV, Elliott RB, \& Emerich DF (2004) Intracerebral transplantation of porcine choroid plexus provides structural and functional neuroprotection in a rodent model of stroke. Stroke, 35(9), 2206-2210.

[8] Brolongan CV, Skinner SJ, Geaney M, Vasconcellos AV, Elliott RB, \& Emerich DF (2004) Neuroprotection by encapsulated choroid plexus in a rodent model of Huntington's disease. Neuroreport, 15(16), 2521-2525.

[9] Emerich DF, Thanos CG, Goddard M, Skinner SJ, Geany MS, Bell WJ, Bintz B, Schneider P, Chu Y, Babu RS, Borlongan CV, Boekelheide K, Hall S, Bryant B, \& Kordower JH (2006) Extensive neuroprotection by choroid plexus transplants in excitotoxin lesioned monkeys. Neurobiol Dis, 23(2), 471-480.

[10] Skinner SJM, Lin H, Geaney MS, Gorba T, Elliott RB, \& Tan PL (2011) Restoration of motor control and dopaminergic activity in Parkinsonian rats by nigrostriatal implants of encapsulated porcine choroid plexus cells. Regen Med, 6(3), 319-326.

[11] Kunishio K, \& Haber SN (1994) Primate cingulostriatal projection: Limbic striatal versus sensorimotor striatal input. $J$ Comp Neurol, 350(3), 337-356.

[12] Parent A, \& Hazarati LN (1995) Functional anatomy of the basal ganglia. I. The cortico-basal ganglia-thalamo-cortical loop. Brain Res Brain Res Rev, 20(1), 91-127.

[13] Villalba RM, Lee H, \& Smith Y (2009) Dopaminergic denervation and spine loss in the striatum of MPTP-treated monkeys. Exp Neurol, 215(2), 220-227.

[14] Lynd-Balta E, \& Haber SN (1994) The organization of midbrain projections to the ventral striatum in the primate. Neuroscience, 59(3), 609-623.

[15] Jan C, Pessiglione M, Tremblay L, Tande D, Hirsch EC, \& Francois C (2003) Quantitative analysis of dopaminergic loss in relation to functional territories in MPTP-treated monkeys. Eur J Neurosci, 18(7), 2082-2086.

[16] Mounayar S, Bouldet S, Tandé D, Jan C, Pessiglione M, Hirsch EC, Féger J, Savasta M, François C, \& Tremblay L (2007) A new model to study compensatory mechanisms in MPTP-treated monkeys exhibiting recovery. Brain, 130(11), 2898-2914.

[17] Kish SJ, Shannak K, \& Hornykiewicz O (1988) Uneven pattern of dopamine loss in the striatum of patients with idiopathic Parkinson's disease. Pathophysiologic and clinical implications. N Engl J Med, 318(14), 876-880.

[18] Fearnley JM, \& Lees AJ (1990) Striatonigral degeneration. A clinicopathological study. Brain, 113(6), 1823-1842.

[19] Liu S, Wang W, Yu X, Mao J, \& Rong P (2006) Establishment of hemi-parkinsonism model of rhesus monkey induced by MPTP. Chin J Med Imaging Technol, 22(3), 353-356.

[20] Wolters EC, Kebabian JC, Guttman M, Mak E, Pate BD, \& Calne DB (1988) A new device for the quantitative assessment of dopaminergic drug effects in unilateral MPTP-lesioned monkeys. Neurosci Lett, 95(1-3), 257-261.

[21] Grondin R, Zhang Z, Ai Y, Ding F, Walton AA, surgener SP, Gerhardt GA, \& Gash DM (2008) Intraputamenal infusion of exogenous neurturin protein restores motor and dopaminer- gic function in the globus pallidus of MPTP-lesioned rhesus monkeys. Cell Transaplant, 17(4), 373-381.

[22] Takagi Y, Takahashi J, Saiki H, Morizane A, Hayashi T, Kishi Y, Fukuda H, Okamoto Y, Koyanagi M, Ideguchi M, Hayashi H, Imazato T, Kawasaki H, Suemori H, Omachi S, Iida H, Itoh N, Nakatsuji N, Sasai Y, \& Hashimoto N (2005) Dopaminergic neurons generated from monkey embryonic stem cells function in a Parkinsonian primate model. J Clin Invest, 115, 102-109.

[23] Clarke CE, Boyce S, Robertson RG, Sambrook MA, \& Crossman AR (1989) Drug-induced dyskinesia in primates rendered hemiparkinsonian by intracarotid administration of 1-methyl-4-phenyl-1,2,3,6-tetrahydropyridine (MPTP). J Neurol Sci, 90(3), 307-314.

[24] Wynyard S, Grakavenko O, \& Elliot R (2011) Multiplex high resolution melting assay for estimation of Porcine Endogenous Retrovirus (PERV) relative gene dosage in pigs and detection of PERV infection in xenograft recipients. $J$ Virol Methods, 175(1), 95-100.

[25] Langston JW, Forno LS, Rebert CS, \& Irwin I (1984) Selective nigral toxicity after systemic administration of 1-methyl4-phenyl-1,2,5,6-tetrahydropyrine (MPTP) in the squirrel monkey. Brain Res, 292(2), 390-394.

[26] Oiwa Y, Eberling JL, Nagy D, Pivirotto P, Emborg ME, \& Bankiewicz KS (2003) Overlesioned hemiparkinsonian non-human primate model: Correlation between clinical, neurochemical and histochemical changes. Front Biosci, 8, a155-a166.

[27] Blesa J, Phani S, Jackson-Lewis V, \& Przedborski S (2012) Classic and new animal models of Parkinson's disease. J Biomed Biotechno, 2012, 845618.

[28] Elsworth JD, Taylor JR, Sladek JR, Jr, Collier TJ, Redmond DE, Jr, \& Roth RH (2000) Striatal dopaminergic correlates of stable parkinsonism and degree of recovery in old-world primates one year after MPTP treatment. Neuroscience, $\mathbf{9 5}(2)$, 399-408.

[29] Eidelberg E, Brooks BA, Morgan WW, Walden JG, \& Kokemoor RH (1986) Variability and functional recovery in the N-methyl-4-phenyl-1,2,3,6-tetrahydropyridine model of parkinsonism in monkeys. Neuroscience, 18(4), 817-822.

[30] Taylor JR, Elsworth JD, Roth RH, Sladek JR Jr, \& Redmond DE (1997) Severe long-term 1-methyl-4-phenyl-1,2,3,6tetrahydropyridine-induced parkinsonism in the vervet monkey (Cercopithecus aethiops sabaeus). Neuroscience, 81(3), 745-755.

[31] Bergman H, RazA, Feingold A, Nini A, Nelken I, Hansel D, Ben Pazi H, \& Reches A (1998) Physiology of MPTP tremor. Mov Disord, 13(Suppl 3), 29-34.

[32] Miller JM, \& McAllister JP II. (2007) Reduction of astrogliosis and microgliosis by cerebrospinal fluid shunting in experimental hydrocephalus. Cerebrospinal Fluid Res 4, 5.

[33] Giladi N. (2002) Assessment of Gait. In: Factor SA, Weiner WJ, editors. Parkinson's Disease: Diagnosis and Clinical Management. New York: Demos Medical Publishing http://www.ncbi.nlm.nih.gov/books/NBK27448/

[34] Willis GL, \& Donnan GA (1987) Histochemical, biochemical and behavioural consequences of MPTP treatment in C-57 black mice. Brain Res, 402(2), 269-274.

[35] Zhang WQ, Tilson HA, Nanry KP, Hudson PM, Hong JS, \& Stachowiak MK (1988) Increased dopamine release from striata of rats after unilateral nigrostriatal bundle damage. Brain Res, 461(2), 335-342.

[36] Altar CA, \& Marien MR (1989) Preservation of dopamine release in the denervated striatum. Neurosci Lett, 96(3), 329334. 
[37] Zigmond MJ, Berger TW, Grace AA, \& Stricker EM (1989) Compensatory responses to nigrostriatal bundle injury. Studies with 6-hydroxydopamine in an animal model of parkinsonism. Mol Chem Neuropathol, 10(3), 185-200.

[38] Abercrombie ED, Bonatz AE, \& Zigmond MJ (1990) Effects of L-dopa on extracellular dopamine in striatum of normal and 6-hydroxydopamine-treated rats. Brain Res, 525(1), 36-44

[39] Robinson TE, Yew J, Paulson PE, \& Camp DM (1990) The long-term effects of neurotoxic doses of methamphetamine on the extracellular concentration of dopamine measured with microdialysis in striatum. Neurosci Lett, 110(1-2) 193-198.

[40] Robinson TE, Castañeda E, \& Whishaw IQ (1990) Compensatory changes in striatal dopamine neurons following recovery from injury induced by 6-OHDA or methamphetamine: A review of evidence from microdialysis studies. Can J Psychol, 44(2), 253-275.

[41] Robinson TE, Mocsary Z, Camp DM, \& Whishaw IQ (1994) Time course of recovery of extracellular dopamine following partial damage to the nigrostriatal dopamine system. $J \mathrm{Neu}$ rosci, 14(5 Pt 1), 2687-2696.
[42] Song DD, \& Haber SN (2000) Striatal responses to partial dopaminergic lesion: Evidence for compensatory sprouting. J Neurosci, 20(13), 5102-5114.

[43] Finkelstein DI, Stanic D, parish CL, Tomas D, Dickson K, \& Horne MK (2000) Axonal sprouting following lesions of the rat substantia nigra. Neuroscience, 97(1), 99-112.

[44] Lau YS, \& Fung YK (1986) Pharmacological effects of 1-methyl-4-phenyl-1,2,3,6-tetrahydropyridine (MPTP) on striatal dopamine receptor system. Brain Res, 369(1-2), 311315.

[45] Dubach M, Schmidt R, Kunkel D, Bowden DM, Martin R, \& German DC (1987) Primate neostriatal neurons containing tyrosine hydroxylase: Immunohistochemical evidence. $\mathrm{Neu}$ rosci Lett, 75(2), 205-210.

[46] Tashiro Y, Sugimoto T, Hattori T, Uemura Y, Nagatsu I, Kikuchi H, \& Mizuno N (1989) Tyrosine hydroxylase-like immunoreactive neurons in the striatum of the rat. Neurosci Lett, 97(1-2), 6-10. 